\title{
The direct generalized predictive position control of USM
}

\author{
Juanping Zhao ${ }^{1, \text { a }}$, Gaoli Chen ${ }^{1, b}$ \\ 'Zhengzhou University of Industry Technology, Henan 450000, China \\ azjptiming@foxmail.com, bamy000@163.com
}

Keywords: Ultrasonic motor, generalized predictive control, Hammerstein model.

\begin{abstract}
Ultrasonic motor has obvious nonlinear and time-varying characteristics, because of its special structure and operating mechanism. So that the control strategy of ultrasonic motor cannot be designed according to traditional electromagnetic motor. It spurs people continually to de research for proper control methods, continually to improve USM's control effect. On the basis of USM's nonlinear Hammerstein model to research on the nonlinear position control strategy of USM. The inversion of the nonlinear part is done, as part of the controller, to compensate the nonlinear part of motor' $\mathrm{s}$ characteristic. Then design the direct generalized predictive control strategy for the remained linear part of motor to realize effective position control of USM. It can fully verify the efficiency of this control strategy by experimental results.
\end{abstract}

\section{Introduction}

With the continuous development of science and technology, industrialization gradually becomes popular and computer technology becomes more and more important in life and production. Because of light weight, small volume, low speed but high torque, not affected by electromagnetic interference, low noise in operation, etc, the field of Ultrasonic motor's employment is expending. Meanwhile, because of the working environment and requirement, the USM with stability, high efficiency and high precision is needed. However, because of USM's paticular operational mechanism, there has twice energy conversion process and the piezoceramic material is easily affected by temperature. The complex relationship of USM's inner state variables, makes USM present obvious nonlinear and time-varying characteristics. It makes the use and control of USM have some limitation. For the control of nonlinear system, there is no unified, effective and blanket theory, just some strategies for the specific problems.

For the generalized predictive self-tuning control of nonlinear system, a lot of researches are made by Chinese and foreign scientists. But it just limit to the control of USM. For the nonlinear and time-varying characteristicsof USM, there is few research to obtain the precise control of speed or position. In this paper, on the basis of the nonlinear Hammerstein model of USM, the direct generalized predictive control method is used to control the position of USM. The result of experiment shows that, the direct generalized predictive control method makes the nonlinear system of USM has good tracking effect and good robustness. It has varified the validity of the control thought by experiments.

\section{The design of self-adaption position controller for USM's nonlinear Hammerstein model}

For the nonlinear Hammerstein model of USM, the static nonlinear part and the dynamic linear part are in series to form the nonlinear model. For the nonlinear part of USM, the inverse of it would be established to counteract the nonlinearity of the system. So that, the model of motor would be a linear system. Then, the controller could be designed according to the linear control theory. Thus, the nonlinear control system of USM is designed as the structure of Fig.2.

The nonlinear part of the nonlinear Hammerstein model has been identified by BFO as the follow:

$$
x(k)=F(u(k))=8.2278+0.0727 u(k)
$$

The linear part is: 




Fig.1 Structure of the nonlinear Hammerstein model of USM and its controller

$$
\frac{y\left(z^{-1}\right)}{x\left(z^{-1}\right)}=\frac{-4.6964+4.6945 z^{-1}}{1-2.1069 z^{-1}+1.1061 z^{-2}}
$$

As shown in Fig. $1, F^{-1}$ is used to present the inverse of the nonlinear part. Because the nonlinear part of model is a linear ploynomial, the process of inverse becomes direct and easy. So the inverse of Eq.(1) could be:

$$
u(k)=-113.175+13.755 x(k)
$$

\section{The design of control strategy by using direct generalized prediction.control algorithm}

By BFO algorithm, the linear part of USM's ninlinear Hammerstein model is identified as Eq.(2).

From Eq.(2) we can get

$$
A\left(z^{-1}\right) y(k)=B\left(z^{-1}\right) x(k)
$$

Here,

$$
\begin{gathered}
A\left(z^{-1}\right)=1-2.1069 z^{-1}+1.1061 z^{-2} \\
B\left(z^{-1}\right)=-4.6964+4.6945 z^{-1}
\end{gathered}
$$

Becasuse the algorithm of generalized prediction is using the controlled autoregressive moving average model to be the predictive model. Thus, Eq.(3) should be converted to the controlled autoregressive moving average model.

The form of the controlled autoregressive moving average model is:

$$
A\left(z^{-1}\right) y(k)=B\left(z^{-1}\right) x(k)+C\left(z^{-1}\right) \varepsilon(\mathrm{k}) / \Delta
$$

Here, $A\left(z^{-1}\right)$ is the polynomical with $m$ order 、 $B\left(z^{-1}\right)$ is the polynomical with $n$ order, $C\left(z^{-1}\right)$ is always set 1 to simplify the algorithm. In addition, the difference factor $\Delta=1-z^{-1}, x(k)$ is the input variable of USM system, $y(k)$ is the output variable of USM system, $\varepsilon(k)$ is the white noise. Eq.(6) can be reduced to

$$
\bar{A} y(k)=B\left(z^{-1}\right) \Delta x(k)+C\left(z^{-1}\right) \varepsilon(k)
$$

Here,

$$
\begin{gathered}
\Delta x(k)=x(k)-x(k-1) \\
\bar{A}=\left(1-z^{-1}\right) A\left(z^{-1}\right)=1+\left(a_{1}-1\right) z^{-1}+\left(a_{2}-a_{1}\right) z^{-2}+\ldots+\left(a_{m}-a_{m-1}\right) z^{-m}-a_{m} z^{-(m+1)} \\
\bar{a}_{m}=a_{m}-a_{m-1}, \quad \bar{a}_{m+1}=a_{m}, \quad \text { so } \\
\bar{A}=1+\bar{a}_{1} z^{-1}+\ldots+\bar{a}_{m} z^{-m}+\bar{a}_{m+1} z^{-(m+1)}
\end{gathered}
$$

Make $a_{0}=1, \quad \bar{a}_{m}=a_{m}-a_{m-1}, \bar{a}_{m+1}=a_{m}$, so

From the above, the nonlinear generalized predicitive model of USM could be:

$$
\begin{aligned}
y(k)=3.1069 y(k-1)-3.2130 y(k-2)+1.1061 y(k-3) \\
-4.6964 x(k)+9.3909 x(k-1)-4.6945 x(k-2)
\end{aligned}
$$


By the property of the control theory, the flowchart of USM's direct generalized predictive position control algorithm is designed as Fig.2 shown:

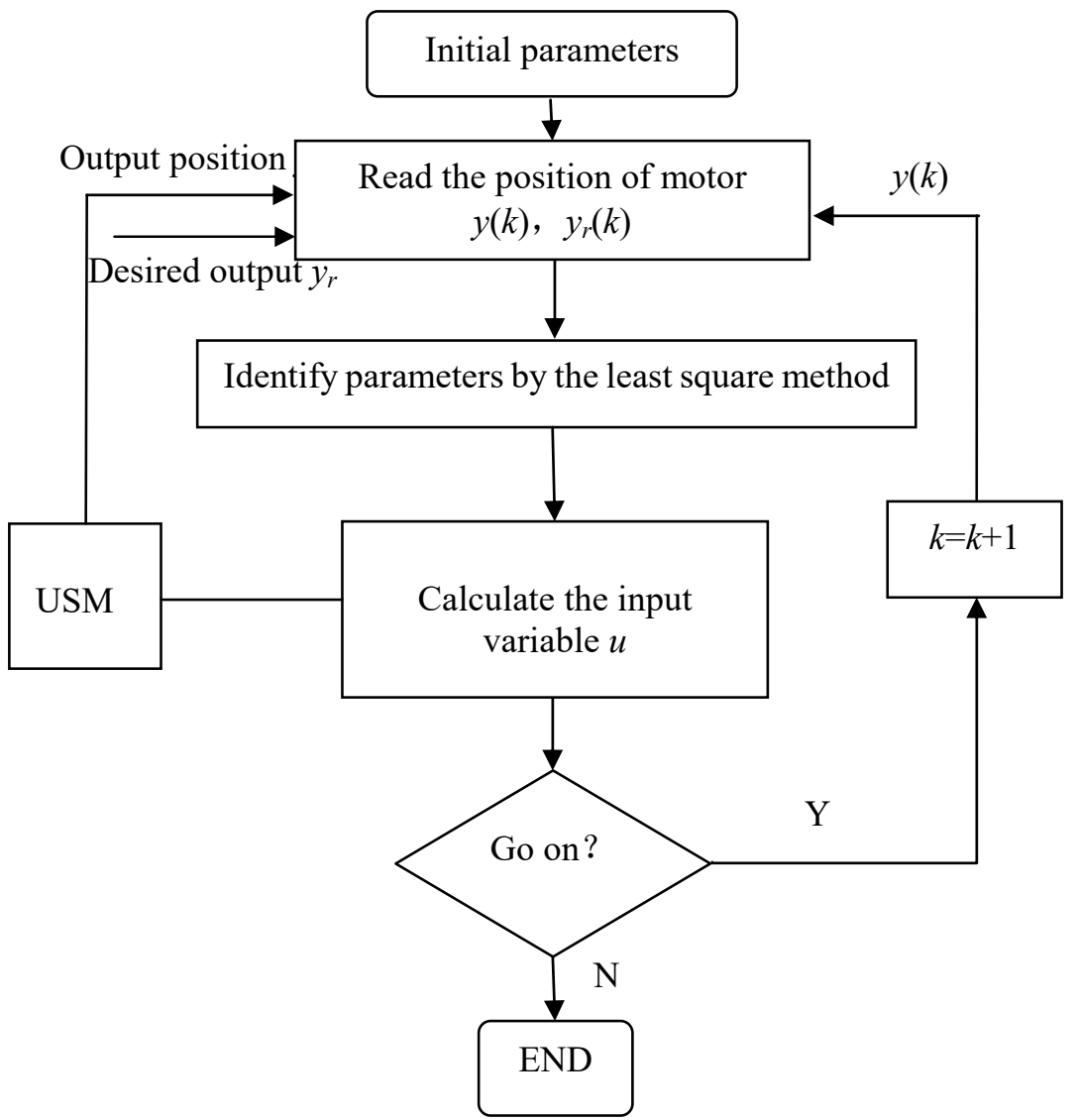

Fig.2 Flowchart of USM's generalized predictive control algorithm When the parameters are adjusted as Tab.1 shown, the contrl effect shows good.

Tab.1 Parameters of Control

\begin{tabular}{ccc}
\hline Parameters & $\begin{array}{c}\text { Initial } \\
\text { value }\end{array}$ & $\begin{array}{c}\text { Value after } \\
\text { adjustment }\end{array}$ \\
\hline Predictive length $N$ & 4 & 5 \\
Control length $N_{u}$ & 1 & 1 \\
Forgetting factor of the LSM $\lambda$ & 1 & 1 \\
Diffusion coefficient $\alpha$ & 0.8 & 0.15 \\
Control weight constant $r_{j}$ & 0.2 & 265 \\
\hline
\end{tabular}

The control effect as Fig.3 shows:

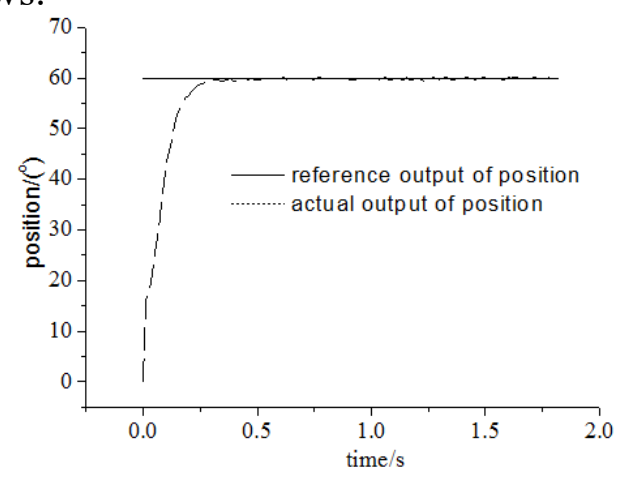

Fig. 3 Output of position $\left(y_{r}=60^{\circ}\right)$ 


\section{Summary}

Experimental result shows that, for the Hammerstein model of USM, the general predictive control method can get good tracking effect. It verified the validity of the control method.

\section{References}

[1]Mohammadreza Ahmadi, Hamed Mojallali, and Mohammad Hossein Fotovvati. Predictive Control of Travelling Wave Ultrasonic Motors using Neural network[C]. 2nd Power Electronics, Drive Systems and Technologies Conference, Tehran: IEEE, 2011: 256-261.

[2]Nooshin Bigdeli, Mohammad Haeri. Simplified modeling and generalized predictive position control of an ultrasonic motor[J]. 2005, 44(2): 273-282.

[3]Hu Zhiqiang, Hu Bo, Liu Yong, Wang Shigang, Wang Ying, Liu Siming. The Predictive Control for a Class of Nonlinear Systems Based on Dynamic Approximate Hammerstein Model[C]. $201231^{\text {st }}$ Chinese Control Conference, Hefei: IEEE, 2012: 4218-4221.

[4]The Model-Free Direct Adaptive Generalized Predictive Control Approach of Permanent Magnet Linear Motor[C]. International Conference on Electrical Machines and Systems, Tokyo: IEEE, 2009: $1-5$. 